\title{
Response of carbon nanotube film transistor to the $\mathrm{THz}$ radiation
}

\author{
V. V. Belosevich ${ }^{1}$, I. A. Gayduchenko ${ }^{2}$, N. A. Titova ${ }^{2}$, E. S. Zhukova ${ }^{1}$, G. N. Goltsman ${ }^{2}$, G. \\ E. Fedorov ${ }^{1}$
}

${ }^{1}$ Moscow Institute of Physics and Technology, Moscow, Russia, vsevolod.belosevich@ phystech.edu ${ }^{2}$ Moscow State Pedagogical University, Moscow, Russia

Nowadays radiation in teraherz frequency range has many applications in different areas of science and industry. Despite years of research, the $\mathrm{THz}$ region of electromagnetic spectrum still has lack of sources and detectors. Almost all created high sensitive detectors require cooling to liquid nitrogen or even helium temperatures.

In 1996 M.Dyakonov and M.Shur have shown theoretically [1] that a short channel High Electron Mobility Transistor demonstrate a resonance response to electromagnetic radiation at the plasma oscillation frequencies of the two-dimensional electrons in the device. The described device implements FieldEffect-Transistor (FET) configuration and consists of transport channel framed by two contacts (source and drain) and a top-gate contact. As a consequence of the high plasma wave velocity and small FET dimensions, the plasma wave frequencies are in the terahertz range. The plasma waves are accompanied by a variation of a dipole moment created by charges in the FET channel and the mirror image charges in the gate.

In that view devices based on carbon nanostructures look promising because of operation with zero bias and their high electron drift mobility. In carbon nanonutubes (CNT) it reaches $100^{\prime} 000 \mathrm{~cm} 2 / \mathrm{Vs}$ [2].

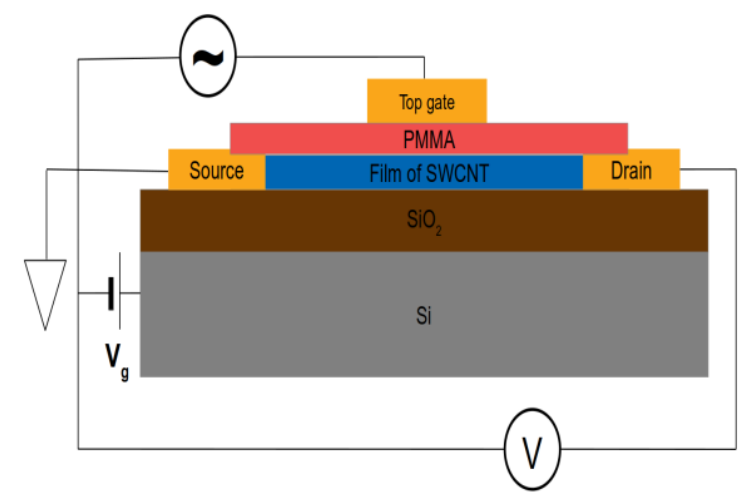

Fig. 1. Scheme of internal structure of the sample

In this work we report on first studies of efficiency of such detectors (Fig.1). Using CVD method we grew our CNT films on oxidized silicon substrate that serves as a back gate of the transistor. After that using photo- and electron- lithography we made Ti/Au contacts and put over a layer of overexposed PMMA resist as dielectric [3]. On the top of the PMMA we plated one more contact as top-gate. Source contact and top gate are part of a broadband log-spiral antenna that couple the radiation (see figure 1). Applying back gate voltage we control concentration and type of charge carriers in transport channel. We observed a strong photoresponse in a broad spectral range from 130 to $900 \mathrm{GHz}$.
In order to charecterize the device we first sweep potential of bottom gate and measure conductance as a function of the back gate voltage applying sourcedrain voltage of $10 \mathrm{meV}$ (see Figure 2). All measurements were carried out at three temperatures (room temperature, temperature of liquid nitrogen, temperature of liquid helium). We further used these dependencies to calculate Seeback coefficient and compare its dependence on $\mathrm{Vg}$ to the gate dependence of the device responsivity (see figure 4 ).

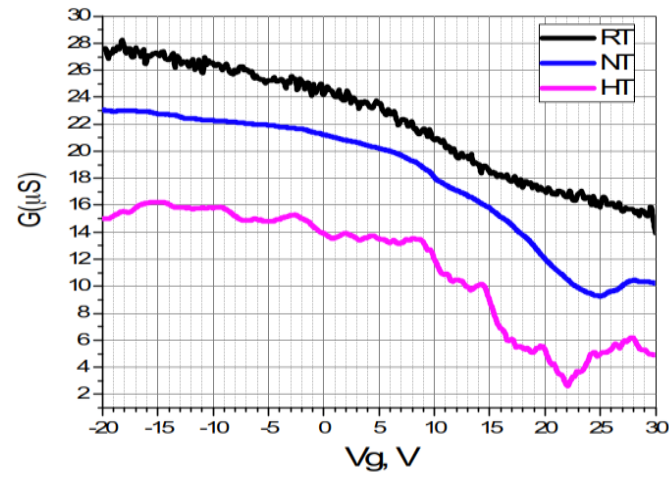

Fig. 2. Transistor characteristic of the tested sample measured at three temperatures $(300 \mathrm{~K}, 77 \mathrm{~K}, 8 \mathrm{~K})$

As seen from the figure 2 the conductance decreases with cooling down, This indicates that there are more semiconducting tubes in the film than metallic, which corresponds with the probabilistic estimate obtained from the conditions on the chirality vector components. [4]

At the lowest temperature on figure $2(8 \mathrm{~K})$ it is possible to observe numerous irregularities. This happens due to different reasons but poor dielectric quality that introduces lots of local potential fluctuations makes this pattern non-reproducible. Charge traps formed by organic molecules of PMMA make noise during the low-temperature experiment.

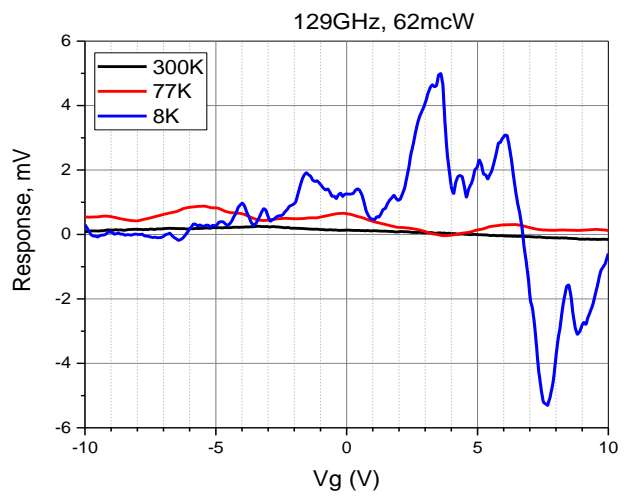

Fig. 3. The photoresponse of the device to $129 \mathrm{GHz}$ radiation at three temperatures $(300 \mathrm{~K}, 77 \mathrm{~K}, 8 \mathrm{~K})$ 
The experimental curves of photoreponse to different temperatures are shown on figure 3 . It can be seen that the response grows up with cooling down the device. On the figure 4 responsivity curves are shown. As can be seen from the figure 4 all curves have a common trend regardless to the frequency of radiation. The main mechanism involved in rectifying the $\mathrm{THz}$ radiation was identified.

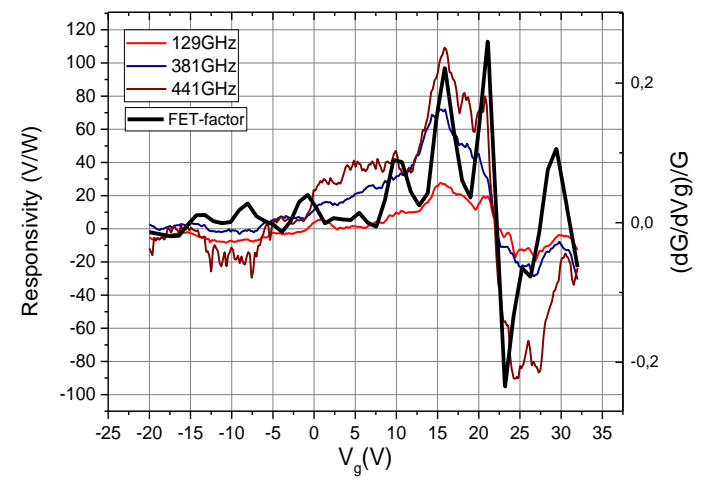

Fig. 4. The responsivity of the device to different frequencies radiation at liquid helium temperature

Thus, we showed that FET device with carbon nanotube film as a transport channel detect $\mathrm{THz}$ radiation in all temperature ranges. Previously CVD graphene device in the Dyakonov-Shur configuration was shown to have 1V/W responsivity [5] and graphene encapsulated between two slabs of hexagonal boron nitride 20V/W [6]. In comparison with this CNT device shows responsivity up to $100 \mathrm{~V} / \mathrm{W}$ to radiation of $\mathrm{THz}$ frequency range. This fact makes CNT devices promising for industry and interesting for scientific research.

\section{References}

1. Dyakonov, M. I., Shur, M. S. Detection, mixing, and frequency multiplication of terahertz radiation by twodimensional electronic fluid // IEEE Transactions on Electron Devices 1996. V. 43. P. 380-387.

2. Dürkop T., Getty S. A. ,Cobas Enrique, and Fuhrer M. S. Extraordinary Mobility in Semiconducting Carbon Nanotubes // Nano Letters 20044 (1), 35-39

3. Sanne A., Movva H. C. P., Kang S., McClellan C., Corbet C. M., Banerjee S. K. Poly(methyl methacrylate) as a self-assembled gate dielectric for graphene field-effect transistors // Appl. Phys. Lett 2014. 104 (8), 83106.

4. Charlier J. C. , Blase X., Roche S. Electronic

and transport properties of nanotubes // B: Reviews of Modern Physics 2007. V.79 Iss 2.

5. Fedorov G.E. , Gaiduchenko I.A., Golikov A.D., Obraztsova E.D., Rybin M.G., Voronov B.M., Coquillat D., Diakonova N., Knap W., Goltsman G.N. Response of Graphene Based Gated Nanodevices Exposed to THz Radiation // EPJ Web of Conferences 103, 10003 (2015).

6. Bandurin D.A., Gaiduchenko I.A., Cao Y., Moskotin M., Principi A., Grigorieva I.V., Goltsman G.N., Fedorov G.E., Svintsov D. Dual origin of room temperature subterahertz photoresponse in graphene field effect transistors // Applied Physics Letters 112, 141101 (2018). 